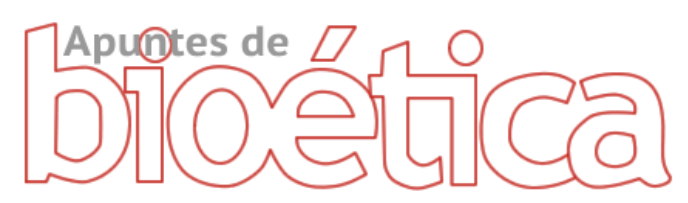

https://doi.org/10.35383/apuntes.v1i1.188

\title{
Estrategias conceptuales para un programa de capacitación para docentes de Educación Básica Regular sobre identidad sexual con enfoque en Bioética personalista
}

\author{
Irene Consuelo Aguilar Valdiviezo ${ }^{1}$
}

\section{INFORMACIÓN DEL ARTÍCULO RESUMEN}

Recibido el 26 de setiembre de 2018

Aceptado el 20 de noviembre de 2018

Palabras claves:

Identidad sexual

Género

Bioética personalista

Sexualidad
El conocimiento de la conformación de la identidad sexual de la persona humana se ha convertido en un importante factor en la formación integral de los docentes de todas las áreas y niveles de la educación básica regular debido a las políticas sobre educación sexual integral que se han introducido en el nuevo currículo nacional con un enfoque de igualdad de género. Por ello, el presente artículo tiene como objetivo abordar las bases teóricas de la identidad sexual para luego dar paso a unas estrategias conceptuales que permitirán, posteriormente, consolidar la propuesta de una capacitación con enfoque bioético personalista. Estas orientaciones resaltan, aunque brevemente, los contenidos que serán abordados en el programa, teniendo en cuenta que con dichos temas los docentes podrán adquirir las competencias necesarias para contribuir en la consolidación de la identidad sexual de sus estudiantes donde se destaque la unicidad de la persona humana como varón o como mujer.

${ }^{1}$ Licenciada en Educación, Coordinadora en la Oficina Diocesana de Educación Católica del Callao, Email: icav0130@gmail.com, Orcid: https://orcid.org/0000-00026760-0524 
Conceptual strategies for a training program for teachers of Regular Basic Education about sexual identity with a focus on Personalistic Bioethics

\section{ABSTRACT}

Keywords:

Sexual identity,

Gender

Personal bioethics

Sexuality
The knowledge of the sexual identity conformation of the human person, has become an important factor in the integral formation of teachers of all areas and levels of Regular Basic Education due to the policies on integral sexual education that have been introduced in the new national curriculum with a focus on gender equality. Therefore, this communication aims to address the theoretical basis of sexual identity and then give way to conceptual strategies that will allow, subsequently, to consolidate the proposal of a training with a personalist bioethical approach. These guidelines highlight, albeit briefly, the contents that will be addressed in the program, taking into account that, with these topics, teachers can acquire the necessary skills to contribute to the consolidation of the sexual identity of their students, where the uniqueness of the human person is highlighted as a man or as a woman.

\section{Introducción}

En el año 2008 la Dirección de Tutoría y Orientación Educativa (DITOE) ${ }^{2}$, presentó los "Lineamientos Educativos y orientaciones pedagógicas para la educación sexual integral: Manual para profesores y tutores de la EBR" (MINEDU, 2008). Con este documento se fundamentaba la obligatoriedad de la enseñanza de la educación sexual integral para los estudiantes de la Educación Básica. Posteriormente en el año 2013 el Ministerio de Educación junto a la Representación de la Unesco en el Perú, publicó “Educación Sexual Integral: Derecho Humano y Contribución a la

\footnotetext{
2 La DITOE es una entidad perteneciente al viceministerio de gestión educativa del Ministerio de Educación. Es la responsable de "normar, planificar, coordinar, asesorar, monitorear y evaluar las acciones de tutoría y orientación educativa" y dentro de las áreas de la tutoría se encuentra, entre otras, la Educación Sexual Integra. Desde esta instancia, el MINEDU, propone las políticas a seguir sobre ESI. También le corresponde las áreas de la promoción para una vida sin drogas y los derechos humanos y la convivencia escolar democrática. MINEDU, (2008).
}

Formación Integral" (MINEDU, 2013), donde señalaba, entre las cuatro condiciones claves para asegurar la efectividad de esta educación en la escuela, el que "esté plenamente integrada en los programas curriculares y que el personal docente esté adecuadamente formado $y$ capacitado para impartirla" (MINEDU, 2013, p. 21). De esta manera se estableció una ruta de implementación de la Educación Sexual Integra en la escuela.

La primera condición de integrar la Educación Sexual Integral en el currículo se oficializó en el año 2016 con la publicación del Nuevo Currículo Nacional bajo Resolución Ministerial N² 281-2016-MINEDU donde reafirma la ESI en todos los niveles de la Educación Básica. Cabe acotar que a diferencia del currículo anterior, publicado en el año 2005 y modificado en el año 2009, este currículo presenta un nuevo enfoque transversal, el de igualdad de género, el 
cual ha sido motivo de discusión entre la sociedad civil peruana porque influye directamente sobre el tipo de educación sexual que se les quiere impartir a los niños y adolescentes ${ }^{3}$.

Frente a esta realidad, la identidad sexual necesita ser conceptualizada desde un enfoque que contemple a la persona humana de manera integral. Para ello nos introduciremos en los conceptos que conforman el concepto de identidad sexual en sus diversas perspectivas. Esto es importante porque requerimos ir sentando las bases de los contenidos para el programa de capacitación sobre la conformación de la identidad sexual desde la bioética personalista. Así mismo daremos una mirada general sobre la formación que se les está impartiendo a los docentes de la Educación Básica Regular sobre Educación Sexual Integral por parte de los entes responsables del MINEDU para, finalmente presentar, de manera tentativa, unas orientaciones para la elaboración del programa de capacitación el cual se consolidará al finalizar la investigación, aún en proceso.

\section{Metodología}

La investigación está basada en el paradigma Teórico y su enfoque de investigación, siguiendo a Sampieri (2014), es el cualitativo. Por otro lado, siguiendo a Bernal (2012), el tipo de investigación es el documental. Para lograr el entendimiento de la conformación de la identidad sexual de la persona humana sin que ésta se sienta afectada en su dignidad, hemos recurrido al tipo de investigación documental ya que analizaremos de manera minuciosa la información bibliográfica sobre los temas que competen a la investigación, como dice el mismo autor: "con el fin de establecer

${ }^{3}$ Burgos nos advierte sobre el peligro de abordar el tema de identidad sexual bajo la mirada de la ideología de género. Véase BURGOS, J M (2011). relaciones, diferencias, etapas, postura o estado actual del conocimiento respecto al tema objeto de estudio" (p. 111); dichos documentos serán analizados para el logro de los objetivos planteados. Las técnicas utilizadas para el recojo de la información ha sido el fichaje bibliográfico, hemerográfico, electrónico, textual, de paráfrasis, de resumen y de comentario; así mismo se ha utilizado las técnicas de análisis documental, la interpretación de textos y el resumen. Por otro lado, con el fin de obtener datos válidos y confiables que nos permitan un adecuado procesamiento y análisis, utilizaremos para nuestro estudio las fichas y los buscadores virtuales como instrumentos de recolección de datos.

\section{Resultados, análisis y discusión}

Nos encontramos realizando un estudio de investigación que se consolidará en la propuesta de un programa de capacitación para docentes de Educación Básica Regular (EBR) sobre identidad sexual con enfoque en bioética personalista. Durante este estudio hemos evidenciado que los conceptos que conforman la identidad sexual, y que serán fundamentales en nuestra programación, han sido objeto de diversas interpretaciones, incluso antagónicas, que han dado origen a eufemismos que van dando paso a una concepción muy distinta a aquello que inicialmente dio origen a dichos términos. Esto ha traído confusión en la sociedad.

Por otro lado, para tener un diagnóstico general sobre la formación de los docentes en temas de identidad sexual creímos conveniente conocer si han recibido algún tipo de capacitación por parte del MINEDU o si existe un plan de acción por parte de alguna entidad responsabilizada para ello. 
Si bien la propuesta del programa es aún incipiente, estos estudios que hasta el momento vamos realizando, y que veremos a continuación, advertirán la pertinencia de una propuesta de capacitación. Por ello ofreceremos, al finalizar, unas orientaciones para la elaboración de dicha propuesta de programación para los docentes.

\section{Eufemismos en torno al concepto de Identidad sexual}

Considerando la pertinencia de las definiciones para el cumplimento del objetivo de este trabajo de investigación, hemos querido considerar estos conceptos solo bajo dos miradas, el de la bioética personalista y el de la ideología de género. De esta manera evidenciaremos con más claridad las posiciones con respecto al concepto.

\section{A. Identidad:}

La bioética personalista define la identidad como "la conciencia que cada persona tiene de sí" (Camps, 2007, p. 110), y que puede entenderse desde dos perspectivas, una la objetiva, es decir aquello que se es dado y la otra, la subjetiva, que es la organización de lo dado según la propia individualidad (Camps, 2007). Para esta toma de conciencia de la propia identidad se requiere de un largo y progresivo proceso donde se combinan varios factores: orgánicos, psicológicos y sociales. Este proceso inicia "en la infancia, adquiere gran importancia en la adolescencia y continúa a lo largo de la vida" (Risco, 2016, págs.154-155). Sin que el individuo pierda la convicción de ser él mismo durante las transformaciones que le son propias en su desarrollo. En oposición a ello la ideología de género conceptualiza la identidad como un proceso de construcción, co-construcción y reconstrucción que se lleva a cabo en las interacciones con las otras personas y por la influencia de los medios de comunicación social (Julio, 2016). Por ese motivo es imposible hablar de una identidad establecida ya que "el sujeto no nace determinado con una identidad última, sino que la identidad es un proceso de constitución nunca acabado" (Navarrete, 2015, p. 477).

\section{B. Sexo}

La bioética personalista nos indica que el sexo es una de las dimensiones complementarias de la identidad sexual de la persona (Aparisi, 2016). Se encuentra determinado biológicamente, constituyendo lo dado, por lo tanto no es posible elegirlo (Elósegui, 2011); así mismo "remite el dato empírico -"recibido y asumido"-, de la dualidad biológica varón/mujer" (Aparisi, 2016, p.26). El sexo es base para la conformación de la identidad sexual, junto con otras dimensiones. Sin embargo la ideología de género le da otro sentido. Sin apartarse del concepto como dato biológico refieren que el sexo o sexo biológico es la base de la asignación del sexo al recién nacido (Julio, 2016), y que junto con otros elementos-género, rol de género, orientación sexual- "emergen a partir de una construcción-producción, social, cultural e histórica y que, por lo tanto, pueden ser deconstruidas para la conformación de un nuevo orden simbólico y social (...)" (Julio, 2016, p.57). Se entiende que, para la ideología de género, el sexo es un accidente más.

\section{Género}

La bioética personalista señala que el género es un aspecto cultural que, junto con el sexo son "dos dimensiones complementarias de la persona, que confluyen en una misma realidad: la identidad sexual del ser humano" (Aparisi, 2016, p.26). Esta conformación de la identidad sexual se manifiesta "en actitudes, 
patrones de comportamiento y atributos de personalidad definidos, principalmente por la cultura en la que el individuo vive, constituyendo así papeles socialmente estereotipados "masculinos" o "femeninos"." (Camps, 2007, p.114). Para la ideología de género, el género tiene mayor relevancia en la definición de la identidad sexual porque no viene de la naturaleza ni está predeterminado al nacer, sino que se construye en cada sociedad (Sheibi y Araujo, 2015); radicalizan "el componente cultural y la autonomía de la voluntad, considerándolos, en definitiva, como los únicos (Aparisi, 2007, p.29), y se ensalza al género porque éste no solo hace referencia a ser varón o ser mujer sino también a toda orientación sexual: heterosexual, homosexual, lesbiana, bisexual y transexual (Miranda, 2012).

\section{Sexualidad:}

La bioética personalista nos indica que la sexualidad es "un elemento básico de la personalidad, un modo propio de ser, de manifestarse, de comunicarse con los otros, de sentir, expresar y vivir el amor humano. $Y$, como los demás elementos constitutivos del ser humano, debe desarrollarse, formarse $y$ educarse" (Jokin, 2012, p. 9). Ésta aún no llega a su máximo desarrollo en el momento del nacimiento sino que se encuentra "abierta a una continua evolución a lo largo de las múltiples etapas por las que atraviesa el individuo" (Vargas, 2013, p. 143). Así mismo la sexualidad humana manifiesta dos novedades: ser principio de pro-creación y ser un "acto exquisito de amor entre un varón y una mujer" (Melendo, 2010). Sin embargo la ideología de género señala que la sexualidad "se refiere a las prácticas sexuales de las personas, ya sean con personas del sexo opuesto (heterosexuales), con personas del mismo sexo (homosexuales), con personas de ambos sexos (bisexuales), con personas trans (omni/pansexuales) o con nadie (asexuales)"
(Sheibi y Araujo, 2015, p.30). Es una práctica sexual sin un fin, diferente a como lo señala la bioética personalista.

\section{E. Identidad Sexual:}

Según los conceptos planteados de identidad, sexo, género y sexualidad hemos podido evidenciar que el enfoque de la bioética personalista y el enfoque de género son antagónicos. Tal vez estén de acuerdo en algunos puntos pero la forma cómo se orientan estos, puede llevar a graves errores. La bioética personalista define la identidad sexual en el contexto del desarrollo sexual refiriéndolo como la "percepción o la íntima convicción que tiene cada uno de pertenecer al sexo masculino o femenino" (Sgreccia, 2014, p.14), y que algunos llaman identidad de género, otros casi lo superponen con el sexo psicológico ya que éste último "constituye una de las últimas etapas en el proceso configurador de la identidad sexual y personal" (Camps, 2007, p.117).

La ideología de género define la identidad sexual como una construcción que si bien parte del dato biológico no es éste el que definirá esta identidad, sino la sociedad. Es así que señalan que "las personas humanas, según el dato biológico del sexo, nacen machos o hembras, pero la sociedad, con su actividad, construye la sexualidad convirtiéndolas en hombres y mujeres, y la cultura engendra las ideas de masculinidad y feminidad" (Trillo, 2009, p.27).

Para entender ello es oportuno indicar que para la ideología de género el carácter sexual es el dato biológico, genético o somático, dado por nacimiento y fuera del ámbito de su libertad y de su identidad. Y para la bioética personalista el carácter sexuado "nos remite a una concepción muy diferente de la integración de la sexualidad de la persona... partir de la persona como 
concepto inicial y, a partir de ella, entender la sexualidad, y no al revés" (Burgos, 2011, p. 417).

\section{Los docentes de la Educación Básica Regular y su formación en identidad sexual}

La formación en identidad sexual forma parte de los contenidos de la Educación Sexual Integral que se promueven en los Lineamientos Educativos y Orientaciones Pedagógicas para la Educación Sexual, citados anteriormente (MINEDU 2008). Esto hace evidente la obligatoriedad por parte de los docentes de empoderarse con los contenidos que van a enseñar, más aún cuando el nuevo currículo nacional contempla, de manera transversal el enfoque de género. No dejemos de considerar que la existencia de eufemismos en torno a la identidad sexual puede llevar a distintas interpretaciones.

Por otro lado el MINEDU es el ente responsable de capacitar a los docentes en todas las áreas curriculares y por consiguiente también, a través de la Dirección de Tutoría y Orientación Educativa en los temas referidos a la identidad sexual en el marco de la Educación Sexual Integral. Esta dirección ha señalado que una de la condiciones que aseguran la efectividad de la Educación Sexual Integral en la escuela es contar con personal docente formado y capacitado por lo que consideran la necesidad de formar nuevos docentes en la especialidad, y capacitar y actualizar a los que se encuentran en servicio (DITOE, 2013). Las acciones asumidas por la Dirección de Tutoría y Orientación Educativa para asegurar esta formación de los docentes podrían verse reflejadas, por un lado, en los insumos elaborados y colocados en la página web del MINEDU, y por otro lado en las jornadas pedagógicas, talleres, seminarios, u otros en las que participan los docentes.
Teniendo en cuenta que el nuevo currículo nacional aún no se ha implementado, salvo a modo de piloto en algunas escuelas del nivel primario, la formación en identidad sexual o en educación sexual integral aún no ha sido asumida por las Direcciones Regionales de Educación o por las Unidades de Gestión Educativa Local o por las mismas Instituciones Educativas de manera oficial, salvo en las áreas donde sí se encuentra integrada como lo son Ciencia, Tecnología y Ambiente, Persona, familia y relaciones humanas, y Tutoría ${ }^{4}$.

La investigación realizada por Guttmacher Institute $^{5}$ junto con la Universidad Cayetano Heredia sobre la aplicación de las políticas en Educación Sexual en la escuela, concluyen que existe poca voluntad política para la implementación de los lineamientos del 2008, que ha dado como fruto la insuficiente capacitación de los docentes en ESI. Al respecto refiere:

El Estado no ha dado un apoyo adecuado a la educación sexual. En el 2008, el MINEDU promulgó los "Lineamientos Educativos y Orientación Pedagógica para la Educación Sexual Integral," pero su implementación ha sido muy débil por falta de voluntad política, lo que se refleja en falta de presupuesto para capacitación de profesores e implementación de sistemas de evaluación y monitoreo. (Instituto Guttmacher, 2017, p.29)

Además de considerar la falta de apoyo del MINEDU también acusa a los mismos docentes de impartir una ESI basada en sus propios ideales de sexualidad o inducidos por motivos religiosos

\footnotetext{
${ }^{4}$ Estas áreas corresponden a las propuestas por el Diseño Curricular Nacional de la Educación Básica Regular, cuya vigencia terminará este año 2018 para dar paso al nuevo currículo nacional a partir del año 2019, cuya implementación se dará según culmine el juicio al cual ha sido sometido este documento por parte de los padres de familia que consideran la existencia de una ideologización de la sexualidad. Algunas de estas áreas ya no han sido contempladas en el nuevo currículo o han sido fusionadas en una sola área.

${ }^{5}$ Guttmacher Institute es una institución sin ánimo de lucro que promueve los derechos de la salud pública reproductiva y por ende el derecho al aborto. Cfr. www, guttmacher.org/es
} 
(Instituto Guttmacher, 2017). Es por ello que ven con preocupación la reacción tenida frente al enfoque de género en el nuevo Currículo indicando que "se ha iniciado una campaña conservadora desde las Iglesias evangélicas, apoyada por la Iglesia católica, que pretende retirar el enfoque de género y contenidos de sexualidad de este nuevo documento" (Instituto Guttmacher, 2017, p. 24), pero al mismo tiempo resaltan como exitosa la defensa que, del nuevo currículo nacional viene haciendo el MINEDU ante el reclamo "de algunos sectores de Iglesias evangélicas y de la Iglesia católica [que] cuestionaron duramente la propuesta de ESI como política pública y el empleo del enfoque de género en el currículo (Instituto Guttmacher, 2017, p. 5).

Por ahora el MINEDU brinda temas referidos a la educación sexual integral para los docentes mediante los link de acceso al repositorio de insumos del MINEDU. Estos materiales educativos, algunos autoformativos, permiten al docente introducirse en la ESI. También puede acceder a sesiones ya elaboradas que facilitan su aplicación directa en el aula.

Bajo la orientación de estos lineamientos la DITOE, con el fin de empoderar a los docentes en los temas de la ESI, ofreció una Propuesta para la formación de docentes en Educación Sexual Integral, donde indicaban que "la capacitación permanente de las y los docentes en servicio adquiere trascendencia y sentido en la Educación Sexual Integral, pues contribuye a desarrollar competencias claves para la vivencia de una sexualidad plena, responsable y saludable en las y los estudiantes" (MINEDU, 2013, p. 7). Sin embargo, esta información no ha llegado a todos los docentes del Perú.

Frente a esta falta de capacitación de los docentes hay instituciones particulares, que se encuentran dando pasos agigantados para revertir esta situación. Muchas de estas instituciones forman parte de la "Alianza por la Educación Sexual Integral" ${ }^{\prime 6}$ cuyo compromiso es impulsar una política pública multisectorial en Educación Sexual Integral en el Perú con un enfoque de género. Entre estas instituciones tenemos a INPPARES ${ }^{7}$ y la ONG Manuela Ramos ${ }^{8}$.

Podemos concluir que la formación de los docentes sobre temas de identidad sexual es incipiente ya que no han sido capacitados al respecto. Pero se espera que a partir de la implementación del nuevo currículo, posiblemente en el 2019, se empiece a capacitar a los docentes sobre esta materia. Por el momento los alcances que el MINEDU ha presentado a docentes y padre de familia, ha consistido en materiales educativos sobre identidad sexual, y cartillas, afiches, trípticos, para explicar el enfoque de igualdad de género ${ }^{9}$, y el tema central sobre el cual se ha concentrado la tutoría es sobre la prevención de las drogas, bajo la orientación de la Comisión Nacional para el Desarrollo y Vida sin Drogas (DEVIDA), desde hace dos años aproximadamente hasta la fecha.

\footnotetext{
${ }^{6}$ Esta alianza nace en el año 2006 en el marco del proyecto "iSí podemos! Realizado por INNPARES entre los años 2006-2008. Cfr. https://www.inppares.org/sipodemos La conforman, entre otras, las siguientes instituciones: Movimiento Manuela Ramos, INPPARES, APROPO, Instituto de Educación y Salud - IES, Asociación Kallpa, Católicas por el Derecho a Decidir Perú, CEPESJU, Amnistía Internacional, Red Interquórum Sociedad Peruana de Adolescencia y Juventud - SPAJ, Centro de la Mujer Flora Tristán. Ver:

https://es.slideshare.net/INPPARESinforma/triptico-de-la-alianza-si-podemos

${ }^{7}$ Esta entidad llevó a cabo, entre los años 2006 y 2008, un proyecto llamado “iSí podemos! en el que desarrolló acciones para introducir en la agenda pública y política, la demanda de una educación sexual integral. Mediante este proyecto, la misma institución señala que influyó en la DITOE para concretizar acuerdos referidos a este tema y así mismo involucró a docentes y estudiantes que permitieron visibilizar y validar la propuesta al Estado para la creación e implementación de una política pública en Educación Sexual Integral. Cabe señalar que esta institución fue también una de las que participó en la elaboración de los lineamientos... Cfr. https://www.inppares.org/sipodemos

8 Desde un enfoque de género se encuentra ejecutando el programa "Generando modelos y cambios intergeneracionales para la garantía de los derechos sexuales, derechos reproductivos y la igualdad de género" como una propuesta pedagógica en 14 escuelas de Ayacucho, Lima y Ucayali Cfr. MANUELA RAMOS, 2016. Disponible en Video en https://www.youtube.com/watch?v=SqIS5Cm2DgE https://www.youtube.com/watch?v=pGXx-gZxOWs

${ }^{9}$ Ver en http://www.minedu.gob.pe/curriculo/
} 


\section{Estrategias conceptuales para el Programa de capacitación}

Al finalizar la investigación presentaremos la propuesta del Programa de capacitación para docentes sobre la conformación de la identidad sexual desde el enfoque de la bioética personalista. Para lograr dicho objetivo seguiremos las siguientes pautas:

\section{A. Elaboración de la justificación del programa de capacitación:}

Señalaremos en este punto la pertinencia de un programa de capacitación con un enfoque que contemple a la persona en toda su unicidad, sin parcializaciones en ninguna de sus dimensiones. Esta característica, como se ha observado en los puntos estudiados, la cumple la bioética personalista ya que mira al hombre como un sujeto individual irrepetible, que posee un cuerpo, una psique y un espíritu (Burgos, 2011). Esta visión holística de la persona humana no parcializa la conformación de la identidad sexual en un aspecto sino que a todos les da el mismo valor.

\section{B. Objetivos, general y específicos:}

Versarán en el conocimiento y el análisis de los supuestos teóricos de la bioética personalista que permitan comprender la conformación de la identidad sexual de la persona humana.

\section{Bases legales:}

Es conveniente amparar esta capacitación con las bases legales que sustenten la formación docente en temas referidos a la sexualidad de la persona humana. Para ello señalaremos los artículos correspondientes de los siguiente documentos legales, entre otros.
- Constitución Política del Perú

- Ley general de Educación Ley 28044

- R.D. No 0180-2008-ED

- Otros a citar.

\section{Contenidos.}

Los temas que se impartirán a los docentes estarán relacionados con los contenidos que el MINEDU propone dar a los estudiantes en el área de Educación sexual integral y más aun los que tengan relación directa con la conformación de la identidad sexual. Bajo ese aspecto se reorientarán dichos temas fundamentándolos con la bioética personalista.

Aún se desconoce si el enfoque de igualdad de género se suprimirá del nuevo currículo o si sufrirá alguna modificación, sin embargo no podemos perder de vista que existe un gran número de organizaciones dedicadas a la capacitación docente que lo harán bajo esa perspectiva.

Bajo esas consideraciones, el aporte que la bioética personalista dará a los temas sobre la conformación de la identidad sexual será relevante. Es por ello que se proponen los siguientes contenidos:

\begin{tabular}{|c|c|}
\hline TEMA & CONTENIDO \\
\hline $\begin{array}{c}\text { I } \\
\text { El currículo } \\
\text { nacional y el } \\
\text { enfoque de } \\
\text { igualdad de } \\
\text { género. }\end{array}$ & $\begin{array}{l}\text { Tema introductorio que permitirá a los } \\
\text { docentes tener una visión general sobre la } \\
\text { propuesta del enfoque de igualdad de } \\
\text { género y la pertinencia de considerar el } \\
\text { enfoque de la bioética personalista para } \\
\text { la educación en la conformación de la } \\
\text { identidad sexual de los estudiantes. } \\
\text { - El nuevo currículo nacional y sus } \\
\text { enfoques transversales. } \\
\text { - El enfoque de igualdad de género y los } \\
\text { valores que promueve: lgualdad y } \\
\text { dignidad, Justicia y empatía. } \\
\text { - El enfoque de la bioética personalista, } \\
\text { un aporte a la conformación de la } \\
\text { identidad sexual. }\end{array}$ \\
\hline II & $\begin{array}{l}\text { En este punto desarrollaremos los } \\
\text { conceptos a partir de los fundamentos de }\end{array}$ \\
\hline
\end{tabular}




\begin{tabular}{|c|c|}
\hline $\begin{array}{l}\text { La bioética } \\
\text { personalista } \\
\text { y sus } \\
\text { consideracio }\end{array}$ & $\begin{array}{l}\text { la bioética personalista y que permitirán ir } \\
\text { consolidando un concepto más adecuado } \\
\text { al tema de identidad sexual. Entre otros, } \\
\text { desarrollaremos estos puntos: }\end{array}$ \\
\hline $\begin{array}{l}\text { nes sobre la } \\
\text { sexualidad } \\
\text { humana. }\end{array}$ & $\begin{array}{l}\text { - La sexualidad humana como } \\
\text { fundamento de identidad de la persona } \\
\text { humana. } \\
\text { - La sexualidad expresión de dos } \\
\text { complementarios, hombres y mujeres }{ }^{10} \text {. }\end{array}$ \\
\hline $\begin{array}{l}\text { III } \\
\text { La identidad } \\
\text { sexual: } \\
\text { Eufemismos } \\
\text { en sus } \\
\text { conceptos }\end{array}$ & $\begin{array}{l}\text { Los contenidos de este tema permitirán al } \\
\text { docente discriminar las nociones de los } \\
\text { conceptos a desarrollar en miras de una } \\
\text { adecuada conformación de la identidad } \\
\text { sexual. } \\
\text { a. Identidad } \\
\text { b. Sexo } \\
\text { c. Género } \\
\text { d. Sexualidad }\end{array}$ \\
\hline $\begin{array}{l}\text { IV } \\
\text { Desarrollo } \\
\text { de la } \\
\text { identidad } \\
\text { sexual }\end{array}$ & $\begin{array}{l}\text { En este tema }{ }^{11} \text { los docentes comprenderán } \\
\text { que en cada etapa de la vida, la persona } \\
\text { humana se va identificando sexualmente } \\
\text { hasta llegar a la configuración de su } \\
\text { identidad sexual. Este desarrollo se } \\
\text { contemplará en dos etapas: } \\
\text { a. Infancia } \\
\text { b. Adolescencia }\end{array}$ \\
\hline $\begin{array}{l}\text { V } \\
\text { La propuesta } \\
\text { de la } \\
\text { bioética } \\
\text { personalista } \\
\text { ante el } \\
\text { enfoque de } \\
\text { igualdad de } \\
\text { género }\end{array}$ & 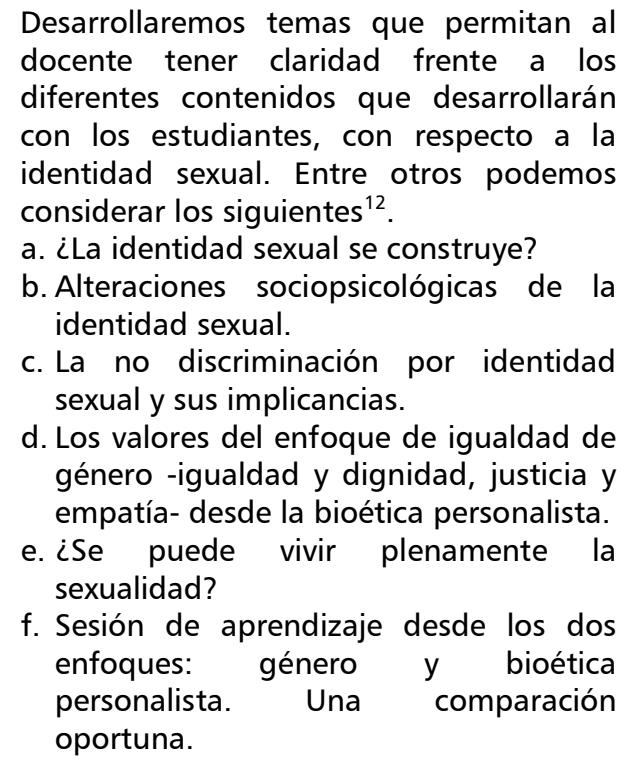 \\
\hline
\end{tabular}

\section{E. Estrategias:}

\footnotetext{
${ }^{10}$ Los autores que ayudarán a centrar este tema son Angela Aparisi, Juan Manuel Burgos.

${ }^{11} \mathrm{El}$ insumo base para trabajar este tema lo tomaremos de la autora Marina Camps en su libro Identidad sexual y derecho: estudio interdisciplinario del transexualismo, p. 117-140.

${ }^{12}$ La orientación conceptual será Marína Camps, p. 141
}

Para el buen desarrollo de las capacitaciones se propondrá una serie de estrategias entre las cuales mencionamos los siguientes:

\section{- Exposiciones \\ - Coloquios \\ - Debates \\ - Simulación de roles \\ - Talleres \\ - Foros de discusión}

\section{F. Medios y materiales:}

Consideraremos locales oportunos para impartir las capacitaciones, y debemos contar con materiales físicos y virtuales pertinentes.

\section{G. Sesiones:}

Para el desarrollo de las sesiones hemos de considerar metodologías oportunas que permitan la participación activa de los docentes, evitando la sobrecarga de exposiciones teóricas que podrían causar un cansancio innecesario. Para lograr ese dinamismo consideraremos los siguientes procesos pedagógicos:

- Recuperación de saberes previos, es decir los conocimientos, habilidades, experiencias y sentimientos que los docentes poseen antes de introducirlos en los contenidos.

- Construcción de nuevos aprendizajes. Estos se construyen sobre los resultados del proceso anterior.

- Talleres de aplicación de lo aprendido.

- Evaluación de los aprendizajes.

Estos elementos, considerados en este esquema, serán validados a medida que desarrollemos la investigación. Por ahora son un 
alcance importante que servirá de base para la propuesta de programación.

\section{Conclusiones}

El enfoque de la bioética personalista con respecto a la identidad sexual permitirá conocer una visión más amplia de su conformación donde se integra a la persona humana en todas sus dimensiones.

La formación en identidad sexual por parte de los docentes de la educación básica regular es incipiente ya que no ha sido priorizada por el Ministerio de Educación como un tema de capacitación. Sí existe un marcado interés de algunas instituciones particulares, ONG, por contribuir con la Educación Sexual Integral, por consiguiente de la identidad sexual, bajo el enfoque de género.

Los docentes de la EBR recibirán capacitaciones sobre temas de identidad sexual con enfoque de igualdad de género a partir de la implementación del nuevo Currículo Nacional en el año 2019. Por ello las estrategias conceptuales propuestas en esta investigación ofrecen una temática que permitirá a los docentes adquirir los conocimientos oportunos para su labor docente en el ámbito de la identidad sexual dentro del marco de la educación sexual integral con un enfoque en bioética personalista.

\section{Bibliografía}

Aparisi, A. (2016). Género y persona. Del posfeminismo de género al modelo de la igualdad en la diferencia. En M. Ledezma (Ed.), Género y justicia: Estudios e investigaciones en el Perú e Iberoamérica. Lima, Perú, Tribunal Constitucional del Perú.
Bernal, C. (2012). Metodología de la investigación, administración, economía, humanidades y ciencias sociales, (3 ed.). Colombia: Pearson.

Burgos, J. M. (2011). Dos formas de afrontar la identidad sexual: personalismo e ideología de género. En Angela Aparisi (Ed.), Personalismo y género. Navarra, España: Asociación Española de Personalismo.

Camps, M. (2007). Identidad sexual y derecho: Estudio interdisciplinario del transexualismo. Navarra, España: Ediciones Universidad de Pamplona.

Elósegui, M. (2011). Tres modelos filosóficos sobre la relación entre sexo y género. En Angela Aparisi (Ed.), Personalismo $y$ género. Navarra, España: Asociación Española de Personalismo.

Jokin De Irala, I. G. (2012). Educación sexual e ideología: jóvenes en la encrucijada. San José, Costa Rica: Promesa.

Julio, C., Kaeuffer, A., Riquelme C., Silva M.P., Osorio, M.R. y Torres, N. (2016). Conocimientos sobre Identidad Sexual de Profesores y Profesoras: ¿Barreras o Facilitadores de Construcción Identitaria? Revista Latinoamericana de Educación Inclusiva, 10(2). 53-71. doi:10.4067/S071873782016000200005

Melendo, T. La belleza de la sexualidad, Recuperado de https://educaenvirtudes.files.wordpress.co m/2010/10/belleza_sexualidad_t_m.pdf

Ministerio de Educación. (2016). Currículo Nacional de la Educación Básica. Recuperado de http://www.minedu.gob.pe/curriculo/pdf/c 
urriculo-nacional-de-la-educacionbasica.pdf

Ministerio de Educación. (2008). Lineamientos educativos y orientaciones pedagógicas para la educación sexual integral: Manual para profesores y tutores de la Educación Básica Regular, Recuperado de http://tutoria.minedu.gob.pe/assets/lineami entos-educativos-y-orientacionespedagogicos-para-educacion-sexualintegral.pdf

Ministerio de Educación. (2013). Educación sexual integral: derecho humano $y$ contribución a la formación integral. Recuperado de http://repositorio.minedu.gob.pe/handle/12 $3456789 / 1463$

Ministerio de Educación. (2013). Propuesta para la formación de docentes en Educación Sexual Integral, (3 ed.), Lima, Perú.

Miranda, M. (2011) Diferencia entre la perspectiva de género y la ideología de género, Dikaion. Recuperado de http://www.scielo.org.co/pdf/dika/v21 n2/v2 1n2a02.pdf

Instituto Guttmacher. (2017). De la Normativa a la Práctica: la Política de Educación Sexual y su Implementación en el Perú. Recuperado de https://www.guttmacher.org/es/report/ politica-de-educacion-sexual-peru

Navarrete-Cazales, Z. (2015) ¿Otra vez la identidad? Un concepto necesario pero imposible, Recuperado de https://www.redalyc.org/pdf/140/14035408 007.pdf

Organización Mundial de la Salud. Género y salud. Recuperado de http://www.who.int/topics/gender/es/
Representación de la Unesco en el Perú. (2013). Educación sexual integral: Derecho Humano y Contribución a la Formación Integral, Lima, Perú: Ministerio de Educación.

Risco, A. (2016) Cuestiones sobre identidad sexual y orientación sexual. En S. Gallardo (Ed.) Persona e identidad, tomo 1. España: Universidad Católica de Ávila.

Sampieri, H. R. y Fernández, C., Baptista, P. (2014). Metodología de la investigación, (6 Ed.), México: Mc Graw Hitl Education.

Scheibe, C. y Araujo, R. (2015). Género, sexo, sexualidades Categorías del debate contemporáneo. Revista Retratos de la Escuela, v. 9, n. 16, ene./jun. 29-46. Recuperado de http//www.esforce.org.br

Sgreccia, E. (2014) Manual de Bioética: Fundamentos y ética biomédica, Vol I, $1^{a}$ ed, $2^{a}$ reimpresión, Madrid, Biblioteca de autores Cristianos.

Vargas, É. Bases de la diferenciación sexual y aspectos éticos de los estados intersexuales (2013), Rev. Reflexiones 92 (1), 141-157. Recuperado de https://revistas.ucr.ac.cr/index.php/reflexion es/article/download/8756/8250 